\title{
Adaptive Friction Compensation in Trajectory Tracking Control of DLR Medical Robots with Elastic Joints
}

\author{
Luc Le-Tien and Alin Albu-Schäffer
}

\begin{abstract}
In this paper we introduce an adaptive control scheme for robots with elastic joints (in particular for the DLR medical robot) in order to increase the positioning accuracy and the performance of control with respect to uncertainties of the parameters of the robot dynamics. In order to design control and analyze system stability a static friction model is applied which describes Coulomb, viscose and load dependent friction. A stability analysis is done for this adaptive control scheme, allowing a Lyapunov based convergence analysis in the context of the nonlinear robot dynamics. Experimental results validate the practical efficiency of the approach.

Index Terms - Adaptive control, adaptive friction compensation, flexible joint robots
\end{abstract}

\section{INTRODUCTION}

Robots with high gear ratio aiming at low own weight and high payload, as the DLR medical robot (Fig. 1, [1]), can be modeled as robots with flexible joints. For these robots the effects of joint friction can strongly influence the system performance. On the other hand, the dynamic parameters of the actuator including friction can strongly vary with time or with temperature, motivating the adaptive control development of this paper.

Different control strategies for elastic joint robots have been developed in the literature. Some methods, such as feedback linearization [2], backstepping [3], or passivity based adaptive control [3], [4] belong to the standard reference and provide control solutions which apply for both regulation and tracking. Singular perturbation controllers [5], [6] can be easily implemented, but are valid only for limited elasticity and lead to some limitations of the overall control bandwidth due to their cascaded nature. Simpler control structures were proposed, such as PD-controller [7] or SISO state feedback controller [8] where for the latter the global asymptotic stability of the closed loop system can be shown.

In order to deal with the strong joint coupling of the DLR medical robot, in [9] a MIMO state feedback controller with full state feedback (motor position, link side torque, as well as their derivatives), gravity and friction compensation was introduced. This controller was already successfully applied to the DLR medical robot. The system stability is derived in analogy to [7] and [8] with a Lyapunov approach.

For the controlled robot the friction compensation is a very basic problem in motion control and therefore there is a huge amount of research literature, out of which only a small fraction can be cited here. The standard approaches

\footnotetext{
Luc Le-Tien is with The Robotics and Mechatronics Center, German Aerospace Center (DLR), Oberpfaffenhofen, D-82234 Wessling, Germany Luc.Le-Tien@dlr.de

Alin Albu-Schäffer is with The Robotics and Mechatronics Center, German Aerospace Center (DLR), Oberpfaffenhofen, D-82234 Wessling, Germany Alin.Albu-Schaefferddlr.de
}

are model based friction compensation with a static friction model [10] or with a dynamic friction model [11]. Since the parameters of friction strongly vary with temperature and with time, model based friction compensation is quite inaccurate. In addition the friction compensation performance with the static friction model is bad with slow velocity because of the chattering phenomena. On the other hand, standard linear techniques such as integrators or disturbance observers [12] are typically used in industrial robotics applications and show good practical performance. For integrators, only local convergence results have been achieved in robotics. Adaptive control techniques in this case can improve control performance with respect to parameter variations.

Most adaptive schemes for robots with flexible joints assume that the link position is measured [13], [14], [15], [16] and neglected effects of friction. In this paper an adaptive control scheme is developed for the DLR medical robot, that takes frictional effects into account. The resulting adaptive controller is experimentally verified and compared with the corresponding model based controller, or rather with the MIMO state feedback controller. The experiments are implemented at the coupled joints 2-3 of the DLR medical robot.

The paper is organized as follows. Section II introduces the robot model. In Sec. III a motion controller is designed based on all known parameters of the robot while Sec. IV summarizes the adaptive controller. Sections III and IV also present the results of the convergence analysis. Sec. IV determines the parametric update law for the adaptive controller. Finally, the obtained performance is verified by experimental tests reported in Sec. V.

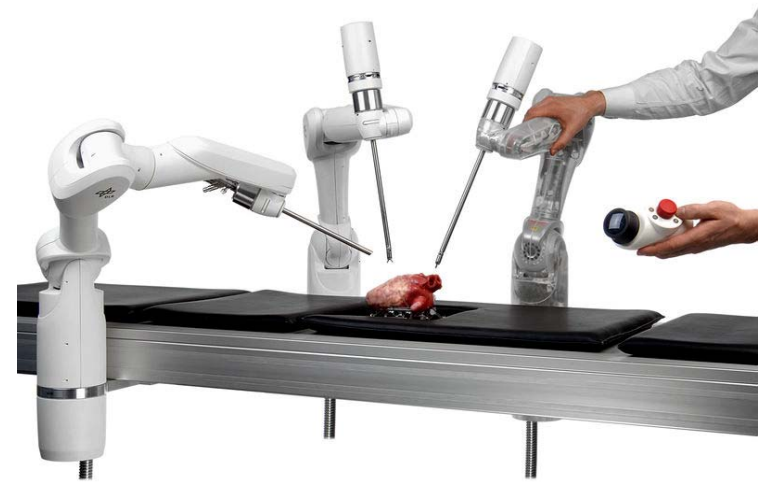

Fig. 1. Setup for using the DLR Medical robot. 


\section{Modeling of the Medical Robot}

\section{A. Dynamics Model}

The DLR medical robot (in Fig. 1) has $n=7$ rotary joints that exhibit considerable elasticity. Apart from the first joint, the following three joint pairs are coupled with differential gears. The following simplified dynamics of the DLR medical robot with flexible joints [9] is described by

$$
\begin{aligned}
u_{m} & =J_{m} \ddot{\theta}_{m}+T^{T} \tau+\tau_{f m} \\
\tau & =M(q) \ddot{q}+C(q, \dot{q}) \dot{q}+g(q)
\end{aligned}
$$

with

$$
\tau=K\left(T \theta_{m}-q\right) .
$$

Therein, $q \in \mathbb{R}^{n}$ and $\theta_{m} \in \mathbb{R}^{n}$ are the link and motor angles, respectively. The elastic torque vector $\tau \in \mathbb{R}^{n}$ is determined by a the linear relationship $\tau=K\left(T \theta_{m}-q\right)$ and is measured by strain gauge based torque sensors. The joint stiffness matrix $K \in \mathbb{R}^{n \times n}$ is symmetric and positive definite and for the DLR medical robot has the following structure

$$
K=\operatorname{diag}\left(K_{1}, K_{2 \_3}, K_{4-5}, K_{6 \_7}\right)
$$

with $K_{1} \in \mathbb{R} \quad, K_{i}=K_{i}^{T} \in \mathbb{R}^{2 \times 2}, \forall i \in\left\{2 \_3,4 \_5,6 \_7\right\}$.

The motor inertia matrix $J_{m} \in \mathbb{R}^{n \times n}$ is diagonal as well. $M(q) \in \mathbb{R}^{n \times n}$ is the mass matrix, $C(q, \dot{q}) \in \mathbb{R}^{n \times n}$ the centrifugal and Coriolis matrix, and $g(q) \in \mathbb{R}^{n}$ the gravity vector of the rigid body model. The control input is the motor torque $u_{m} \in \mathbb{R}^{n} . \tau_{f m} \in \mathbb{R}^{n}$ is the friction torque.

Due to the coupling of the joints through the differential gear (see Fig. 2), motor coordinates, denoted by the subscript $m$, have to be distinguished from the coordinates after the gearbox (or, on the link side), written without subscripts. For example, the motor position $\theta_{m}$ as well as the motor inertia $J_{m}$ are given in motor coordinates, while the joint torque $\tau$ is measured after the gear, in link coordinates. Due to the differential gears, the transformations between motor and link coordinates for positions and torques are given by

$$
\left\{\begin{array}{l}
\theta=T \theta_{m} \\
\tau_{m}=T^{T} \tau
\end{array}\right.
$$

with $\mathrm{T}$ being the transformation matrix

$$
T=\left[\begin{array}{cccc}
1 & \ldots & & 0 \\
& \mathcal{T} & & \\
\vdots & & \mathcal{T} & \vdots \\
0 & \ldots & & \mathcal{T}
\end{array}\right], \forall \mathcal{T}=\left[\begin{array}{rr}
0.5 & 0.5 \\
-0.5 & 0.5
\end{array}\right]
$$

Finally, in order to facilitate the controller design and the stability analysis the following two properties are used

1) The matrix $\dot{M}(q)-2 C(q, \dot{q})$ is skew symmetric and

$$
x^{T}(\dot{M}(q)-2 C(q, \dot{q})) x=0 \quad \forall x, q, \dot{q} \in \mathbb{R}^{n} .
$$

2) The dynamic parameters of the rigid body can be linearized and enable to write

$$
M(q) \ddot{q}+C(q, \dot{q}) \dot{q}+g(q)=Y_{q}(q, \dot{q}, \ddot{q}) \gamma_{q},
$$

where $\gamma_{q} \in \mathbb{R}^{m}$ is the vector of the rigid body parameters and $Y_{q} \in \mathbb{R}^{n \times m}$ is the corresponding regressor matrix of known functions.

\section{B. Friction Model}

In this paper a static friction model is chosen for the control design and the stability analysis. In particular, we consider the following standard friction model containing Coulomb friction, load dependent friction and viscous friction. So the friction model is given by

$$
\tau_{f m}=\left(f_{c}+\mu\left|\tau_{m}\right|\right) \operatorname{sign}\left(\dot{\theta}_{m}\right)+f_{v} \dot{\theta}_{m}
$$

with $f_{c}, f_{v}$ and $\mu$ being the Coulomb, viscous and load dependent coefficient matrices, respectively. $\tau_{m}$ is the joint torque at the motor side. Furthermore the friction model can be described by

$$
\tau_{f m}=Y\left(\dot{\theta}_{m}, \tau_{m}\right) \gamma_{f}
$$

with

$$
\begin{aligned}
& \gamma_{f}= {\left[f_{c_{11}}, \ldots, f_{c_{n n}}, \mu_{11}, \ldots, \mu_{n n},\right.} \\
&\left.f_{v_{11}}, \ldots, f_{v_{n}}\right]^{T} \in \mathbb{R}^{3 n} \\
& Y\left(\dot{\theta}_{m}, \tau_{m}\right)= {\left[\operatorname{diag}\left(\operatorname{sign}\left(\dot{\theta}_{m}\right)\right), \operatorname{diag}\left(\left|\tau_{m}\right| \operatorname{sign}\left(\dot{\theta}_{m}\right)\right),\right.} \\
&\left.\operatorname{diag}\left(\dot{\theta}_{m}\right)\right] \in \mathbb{R}^{n \times 3 n}
\end{aligned}
$$

\section{CONTROL DESIGN With ALL PARAMETERS KNOWN}

In this paper the stiffness $K$ of the robot may be assumed to be known. So the link position $q$ can be calculated by $q=T \theta_{m}-K^{-1} \tau$, when the motor position $\theta_{m}$ and torque $\tau$ are measured. For the controller design, the dynamics (1), (2) is rewritten in terms of link coordinates (after the gearbox) as

$$
\begin{aligned}
u & =J \ddot{\theta}+K(\theta-q)+\tau_{f} \\
\theta & =K^{-1}[M(q) \ddot{q}+C(q, \dot{q}) \dot{q}+g(q)]+q
\end{aligned}
$$

The following tensor transformations have been used

$$
\left\{\begin{array}{l}
u=T^{-T} u_{m} \\
\theta=T \theta_{m} \\
J=T^{-T} J_{m} T^{-1} \\
\tau_{f}=T^{-T} \tau_{f m} .
\end{array}\right.
$$

Notice that $J \in \mathbb{R}^{n \times n}$, the motor inertia matrix written in link coordinates, is positive definite and symmetric, but in general not diagonal.

In order to control the robot to track the desired trajectory $q_{d}$, a joint motion controller is developed based on the rigid body dynamics which generates the desired motor position $\theta_{d}$. Then, based on this desired motor position the suitable

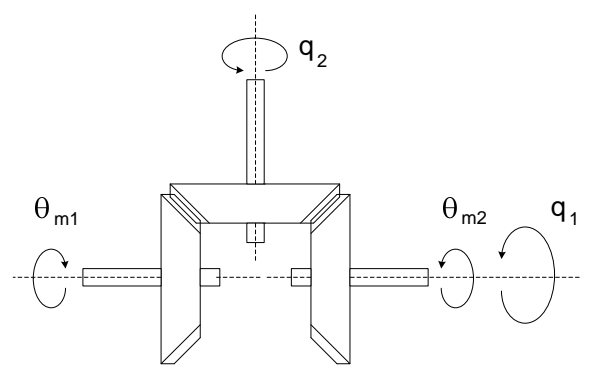

Fig. 2. Differential gear at the DLR Medical Robot. 
control torque $u$ is computed by a motor motion controller so that the motor position can follow the desired motor position $\theta_{d}$, and thus the link position $q$ will follow the desired link position $q_{d}$.

\section{A. Joint Motion Controller}

Let $\lambda_{q}$ be a positive definite and diagonal matrix, and define

$$
\left\{\begin{array}{l}
e_{q}=q_{d}-q \\
v_{q}=\dot{q}_{d}+\lambda_{q} e_{q} \\
s_{q}=v_{q}-\dot{q}=\dot{e}_{q}+\lambda_{q} e_{q} .
\end{array}\right.
$$

The designed motor position $\theta_{d}$ is generated by using the rigid body dynamics [17]

$$
\begin{aligned}
\theta_{d}= & K^{-1}\left[M(q) \dot{v}_{q}+C(q, \dot{q}) v_{q}+g(q)\right] \\
& +K^{-1} K_{q} s_{q}+q
\end{aligned}
$$

where the matrix $K_{q}$ is positive definite and diagonal.

Substituting (13) into equation (10) we obtain the closedloop rigid-body dynamics

$$
\begin{aligned}
e_{\theta} & =\theta_{d}-\theta \\
& =K^{-1}\left[M(q) \dot{s}_{q}+C(q, \dot{q}) s_{q}\right]+K^{-1} K_{q} s_{q} .
\end{aligned}
$$

\section{B. Motor Motion Controller}

Further let us define the tracking errors of motor side

$$
\left\{\begin{array}{l}
e_{\theta}=\theta_{d}-\theta \\
v_{\theta}=\dot{\theta}_{d}+\lambda_{\theta} e_{\theta} \\
s_{\theta}=v_{\theta}-\dot{\theta}=\dot{e}_{\theta}+\lambda_{\theta} e_{\theta} .
\end{array}\right.
$$

with $\lambda_{\theta}$ being a positive definite and diagonal matrix.

To ensure that the motor position $\theta$ converges to the designed motor position $\theta_{d}$, a computed torque like control law is chosen as

$$
u=J \dot{v}_{\theta}+K(\theta-q)+K_{\theta} s_{\theta}+\tau_{f}\left(\dot{\theta}_{m}, \tau_{m}\right)
$$

with the friction torque (11) being $\tau_{f}=T^{-T} \tau_{f m}\left(\dot{\theta}_{m}, \tau_{m}\right)$ and $K_{\theta}$ being a positive definite and diagonal matrix.

Then, substituting (16) into (9), we have the closed-loop of the motor dynamics

$$
J \dot{s}_{\theta}+K_{\theta} s_{\theta}=0 .
$$

\section{Stability Analysis}

For stability analysis the following Lyapunov function candidate was used

$$
V_{1}=\frac{1}{2} s_{q}^{T} M(q) s_{q}+\frac{1}{2} s_{\theta}^{T} J s_{\theta}+e_{\theta}^{T} K_{\theta} \lambda_{\theta} e_{\theta} .
$$

This Lyapunov function contains in addition to the motor and link side kinetic energy also the potential energy related the motor motion controller, and is always positive definite.

The derivative of the Lyapunov function $V_{1}$ along the system trajectories using the equations (17), (15), (14) leads to

$$
\begin{aligned}
\dot{V}_{1}= & s_{q}{ }^{T} M(q) \dot{s}_{q}+\frac{1}{2} s_{q}^{T} \dot{M}(q) s_{q} \\
& +s_{\theta}^{T} J \dot{s}_{\theta}+2 \dot{e}_{\theta}^{T} K_{\theta} \lambda_{\theta} e_{\theta} \\
= & s_{q}^{T} K e_{\theta}-s_{q}^{T} K_{q} s_{q}-e_{\theta}^{T} \lambda_{\theta}^{T} K_{\theta} \lambda_{\theta} e_{\theta}-\dot{e}_{\theta}^{T} K_{\theta} \dot{e}_{\theta} \\
= & -\left[\begin{array}{ll}
s_{q}{ }^{T} & e_{\theta}^{T}
\end{array}\right] H\left[\begin{array}{l}
s_{q} \\
e_{\theta}
\end{array}\right]-\dot{e}_{\theta}^{T} K_{\theta} \dot{e}_{\theta}
\end{aligned}
$$

with the Hessian-matrix

$$
H=\left[\begin{array}{cc}
K_{q} & -\frac{1}{2} K \\
-\frac{1}{2} K & \lambda_{\theta}{ }^{T} K_{\theta} \lambda_{\theta}
\end{array}\right]
$$

Then, the function $\dot{V}_{1}$ is negative definite when the Hessian-matrix $H$ is positive definite or the following condition

$$
\lambda_{\theta}{ }^{T} K_{\theta} \lambda_{\theta}>\frac{1}{4} K^{T} K_{q}^{-1} K
$$

is fulfilled.

Therefore, the system is stable in the sense of the Lyapunov theory because of the functions $V_{1}>0$ and $\dot{V}_{1}<0$. From the closed-loop dynamics (17) and (14), $\dot{s}_{\theta}$ and $\dot{s}_{q}$ are bounded. Since $s_{\theta}$ and $s_{q}$ are bounded and are also uniformly continuous functions, it follows that $\dot{V}_{1}$ is bounded and uniformly continuous function. Therefore,

$$
\lim _{t \rightarrow \infty} s_{\theta}=0 \text {, and } \lim _{t \rightarrow \infty} s_{q}=0
$$

and $e_{\theta} \rightarrow 0, \dot{e}_{\theta} \rightarrow 0, e_{q} \rightarrow 0$, and $\dot{e}_{q} \rightarrow 0$ as $t \rightarrow 0$. Therefore the system is globally asymptotically stable.

\section{AdAptive Control Design}

\section{A. Adaptive Joint Motion Controller}

For unknown parameters the adaptive control law of the designed motor position $\theta_{d}$ is chosen by

$$
\begin{aligned}
\theta_{d}= & K^{-1}\left[\widehat{M}(q) \dot{v}_{q}+\widehat{C}(q, \dot{q}) v_{q}+\widehat{g}(q)\right] \\
& +K^{-1} K_{q} s_{q}+q \\
= & K^{-1} Y_{q}\left(q, \dot{q}, v_{q}, \dot{v}_{q}\right) \widehat{\gamma}_{q}+K^{-1} K_{q} s_{q}+q
\end{aligned}
$$

where $\widehat{M}(q), \widehat{C}(q, \dot{q})$ and $\widehat{g}(q)$ are adapted parameters. Substituting (21) into equation (10), leads to the closed-loop rigid body dynamics

$$
\begin{aligned}
e_{\theta}= & \theta_{d}-\theta \\
= & -K^{-1} Y_{q}\left(q, \dot{q}, v_{q}, \dot{v}_{q}\right) \widetilde{\gamma}_{q} \\
& +K^{-1}\left[M(q) \dot{s}_{q}+C(q, \dot{q}) s_{q}\right]+K^{-1} K_{q} s_{q} .
\end{aligned}
$$

where $\gamma_{q}, \widehat{\gamma}_{q}$ are the dynamic parameters of the rigid body model and their estimates respectively, and $\widetilde{\gamma}_{q}=\gamma_{q}-\widehat{\gamma}_{q}$.

\section{B. Adaptive Motor Motion Controller}

The following adaptive control law for the motor motion control based on the robot dynamics with the static friction model (7) is chosen

$$
\begin{aligned}
u= & \widehat{J} \dot{v}_{\theta}+K(\theta-q)+K_{\theta} s_{\theta} \\
& +T^{-T}\left[\left(\widehat{f_{c}}+\widehat{\mu}\left|\tau_{m}\right|\right) \operatorname{sign}\left(v_{m}\right)+\widehat{f}_{v} v_{m}\right] \\
\equiv & \widehat{J} \dot{v}_{\theta}+K(\theta-q)+K_{\theta} s_{\theta}+T^{-T} Y_{f}\left(v_{m}, \tau_{m}\right) \widehat{\gamma}_{f}
\end{aligned}
$$

with $v_{m}=T^{-1} v_{\theta}$ and $\widehat{\gamma}_{f}$ being the estimates of the friction parameters.

Substituting (23) into equation (9) and utilizing equations (8), (11), (15) yield the closed-loop motor dynamics

$$
\begin{array}{r}
J \dot{s}_{\theta}+K_{\theta} s_{\theta}+T^{-T}\left[Y_{f}\left(v_{m}, \tau_{m}\right)-Y_{f}\left(\dot{\theta}_{m}, \tau_{m}\right)\right] \gamma_{f}= \\
Y_{\theta}\left(\dot{v}_{\theta}\right) \widetilde{\gamma}_{\theta}+T^{-T} Y_{f}\left(v_{m}, \tau_{m}\right) \widetilde{\gamma}_{f}
\end{array}
$$

where $\widetilde{\gamma}_{f}=\gamma_{f}-\widehat{\gamma}_{f}$, and $\widetilde{J}=J-\widehat{J}$, and where

$$
\begin{aligned}
\widetilde{\gamma}_{\theta} & =\left[\widetilde{J}_{11}, \widetilde{J}_{22}, \ldots, \widetilde{J}_{n n}\right]^{T} \in \mathbb{R}^{n} \\
Y_{\theta}\left(\dot{v}_{\theta}\right) & =\operatorname{diag}\left(\dot{v}_{\theta_{1}}, \dot{v}_{\theta_{2}}, \ldots, \dot{v}_{\theta_{n}}\right) \in \mathbb{R}^{n \times n} .
\end{aligned}
$$




\section{Stability Analysis}

Stability analysis of the closed-loop dynamics (22) and (24) can be shown by considering the following Lyapunov function candidate

$$
V=V_{1}+\frac{1}{2} \widetilde{\gamma}_{q}^{T} \Gamma_{q}^{-1} \widetilde{\gamma}_{q}+\frac{1}{2} \widetilde{\gamma}_{\theta}^{T} \Gamma_{\theta}^{-1} \widetilde{\gamma}_{\theta}+\frac{1}{2} \widetilde{\gamma}_{f}^{T} \Gamma_{f}^{-1} \widetilde{\gamma}_{f}
$$

where $\Gamma_{q}, \Gamma_{\theta}$ and $\Gamma_{f}$ are positive definite and diagonal matrices. So this Lyapunov function is always positive definite.

The derivative of the Lyapunov function $V$ along the system trajectories and utilizing equations (22), (24) is given

$$
\begin{aligned}
\dot{V}= & s_{q}{ }^{T} M(q) \dot{s}_{q}+\frac{1}{2} s_{q}{ }^{T} \dot{M}(q) s_{q}+2 \dot{e}_{\theta}^{T} K_{\theta} \lambda_{\theta} e_{\theta} \\
& +s_{\theta}{ }^{T} \dot{s}_{\theta}+\dot{\vec{\gamma}}_{q}^{T} \Gamma_{q}^{-1} \widetilde{\gamma}_{q}+\dot{\tilde{\gamma}}_{\theta}{ }^{T} \Gamma_{\theta}^{-1} \widetilde{\gamma}_{\theta}+\dot{\gamma}_{f}{ }^{T} \Gamma_{f}^{-1} \widetilde{\gamma}_{f} \\
= & s_{q}{ }^{T} K e_{\theta}-s_{q}{ }^{T} K_{q} s_{q}-\dot{e}_{\theta}^{T} K_{\theta} \dot{e}_{\theta}-e_{\theta}{ }^{T} \lambda_{\theta}{ }^{T} K_{\theta} \lambda_{\theta} e_{\theta} \\
& +\left[s_{q}{ }^{T} Y_{q}\left(q, \dot{q}, v_{q}, \dot{v}_{q}\right)+\dot{\tilde{\gamma}}_{q}^{T} \Gamma_{q}^{-1}\right] \widetilde{\gamma}_{q} \\
& \left.+\left[s_{\theta}{ }^{T} Y_{\theta} \dot{v}_{\theta}\right)+\dot{\widetilde{\gamma}}_{\theta}^{T} \Gamma_{\theta}^{-1}\right] \widetilde{\gamma}_{\theta} \\
& +\left[s_{\theta}{ }^{T} T^{-T} Y_{f}\left(v_{m}, \tau_{m}\right)+\dot{\widetilde{\gamma}}_{f}^{T} \Gamma_{f}^{-1}\right] \widetilde{\gamma}_{f} \\
& -s_{\theta}{ }^{T} T^{-T}\left[Y_{f}\left(\dot{\theta}_{m}, \tau_{m}\right)+Y_{f}\left(v_{m}, \tau_{m}\right)\right] \gamma_{f} \\
\equiv & \dot{V}_{1}+\dot{V}_{2}+\dot{V}_{3}
\end{aligned}
$$

with

$$
\begin{aligned}
\dot{V}_{1}= & s_{q}^{T} K e_{\theta}-s_{q}{ }^{T} K_{q} s_{q}-\dot{e}_{\theta}^{T} K_{\theta} \dot{e}_{\theta}-e_{\theta}{ }^{T} \lambda_{\theta}{ }^{T} K_{\theta} \lambda_{\theta} e_{\theta} \\
\dot{V}_{2}= & {\left[s_{q}{ }^{T} Y_{q}\left(q, \dot{q}, v_{q}, \dot{v}_{q}\right)+\dot{\gamma}_{q}^{T} \Gamma_{q}^{-1}\right] \widetilde{\gamma}_{q} } \\
& +\left[s_{\theta}{ }^{T} Y_{\theta}\left(\dot{v}_{\theta}\right)+\dot{\tilde{\gamma}}_{\theta}^{T} \Gamma_{\theta}^{-1}\right] \widetilde{\gamma}_{\theta} \\
& +\left[s_{\theta}{ }^{T} T^{-T} Y_{f}\left(v_{m}, \tau_{m}\right)+\dot{\widetilde{\gamma}}_{f}^{T} \Gamma_{f}^{-1}\right] \widetilde{\gamma}_{f} \\
\dot{V}_{3}= & -s_{\theta}{ }^{T} T^{-T}\left[Y_{f}\left(v_{m}, \tau_{m}\right)-Y_{f}\left(\dot{\theta}_{m}, \tau_{m}\right)\right] \gamma_{f} .
\end{aligned}
$$

From section (III) the function $\dot{V}_{1}$ is positive definite when the condition (20) is fulfilled.

Since the parameter vectors $\gamma_{q}, \gamma_{\theta}$ and $\gamma_{f}$ are constant, the update laws for the estimated robot parameters are chosen

$$
\left\{\begin{array}{l}
\dot{\hat{\gamma}}_{q}=\Gamma_{q}^{T} Y_{q}\left(q, \dot{q}, v_{q}, \dot{v}_{q}\right)^{T} s_{q} \\
\dot{\widehat{\gamma}}_{\theta}=\Gamma_{\theta}^{T} Y_{\theta}\left(\dot{v}_{\theta}\right)^{T} s_{\theta} \\
\dot{\widehat{\gamma}}_{f}=\Gamma_{f}^{T} Y_{f}\left(v_{m}, \tau_{m}\right)^{T} T^{-1} s_{\theta} .
\end{array}\right.
$$

This makes the function $\dot{V}_{2}$ equal to zero.

Further, setting $f_{s}=\left(f_{c}+\mu\left|\tau_{m}\right|\right)$ (and always $f_{s}>0$ ) and from equations (8), (11) and (15) the function $\dot{V}_{3}$ can be rewritten as

$$
\begin{aligned}
\dot{V}_{3}= & -\left(v_{m}-\dot{\theta}_{m}\right)^{T}\left[f_{s} \operatorname{sign}\left(v_{m}\right)+f_{v} v_{m}\right. \\
& \left.-f_{s} \operatorname{sign}\left(\dot{\theta}_{m}\right)-f_{v} \dot{\theta}_{m}\right] \\
=\sum_{i=1}^{n}-f_{v_{i}}\left(v_{m_{i}}\right. & \left.-\dot{\theta}_{m_{i}}\right)^{2}-f_{s_{i}}\left[\left|v_{m_{i}}\right|-v_{m_{i}} \operatorname{sign}\left(\dot{\theta}_{m_{i}}\right)\right] \\
& -f_{s_{i}}\left[\left|\dot{\theta}_{m_{i}}\right|-\dot{\theta}_{m_{i}} \operatorname{sign}\left(v_{m_{i}}\right)\right] \leq 0
\end{aligned}
$$

and is always negative definite.

Thus, the Lyapunov function $V$ is positive definite and $\dot{V}$ is negative definite with the conditions (20) and the adaptive law (28). The system is also stable in sense of the Lyapunov theory. Further, the errors $s_{\theta}$ and $s_{q}$ are uniformly and continuously. Therefore

$\lim _{t \rightarrow \infty} e_{\theta}=0, \lim _{t \rightarrow \infty} \dot{e}_{\theta}=0, \lim _{t \rightarrow \infty} e_{q}=0$, and $\lim _{t \rightarrow \infty} \dot{e}_{q}=0$.

The system is also globally asymptotically stable.

\section{EXPERIMENTS}

In this section the results of two experiments with the controllers of Sec. III and Sec. IV are compared for the coupled joints 2-3 of the DLR medical robot, with

$$
\mathcal{T}=\left[\begin{array}{cc}
-0.5 & 0.5 \\
-0.5 & -0.5
\end{array}\right]
$$

Both axes are controlled to track a desired periodic trajectory (see Fig. 3) with a desired velocity of $[10,14](\mathrm{deg} / \mathrm{s})$. The following gain parameters are chosen in the experiments $\lambda_{q}=\operatorname{diag}(25,25), \lambda_{\theta}=\operatorname{diag}(25,25), K_{q}=\operatorname{diag}(20,20)$, $K_{\theta}=\operatorname{diag}(20,20), \Gamma_{q}=\operatorname{diag}(10,10,10,10), \Gamma_{\theta}=$ $\operatorname{diag}(10,10)$ and $\Gamma_{f}=\operatorname{diag}(30,30,30,30,30,30)$.

In the first experiment, the model based controller with known parameters according to Sec. III is applied in order to show the behavior in terms of the tracking errors. In this experiment it has been assumed that the real friction parameters used for the controller have been identified with a deviation under $5 \%$. Figure 4 shows the position errors of both joints. Another experiment with the model based controller shows that this controlled system is very sensitive with respect to uncertainties of the parameters. So for $30 \%$ error of the friction parameters the system becomes unstable.

For the case of the adaptive controller according to Sec. IV the same desired trajectory is used. The initial values of the estimated friction parameters are $80 \%$ of the identified friction value $f_{c_{\text {init }}}=[8.8,6.8] \mathrm{Nm}, \mu_{\text {init }}=[0.35,0.06]$, and $f_{v_{\text {init }}}=[7.0,10.8] \mathrm{Nms} / \mathrm{rad}$. The estimates of the static friction parameters are shown in Fig. 6, Fig. 7, and Fig. 8. The position errors of both joints are shown in Fig. 5.

It can be observed from the tracking errors that the adaptive controller with friction compensation is considerably superior in performance with respect to the model based controller. Those tracking errors were improved significantly due to the adaption.

Further, this result is compared with the case of MIMO state feedback controller [9] which has the linear control law

$$
\begin{aligned}
u= & K_{P} e_{\theta}-K_{D} \dot{\theta}+K_{T} K^{-1}\left(g\left(q_{d}\right)-\tau\right)-K_{S} K^{-1} \dot{\tau} \\
& +g\left(q_{d}\right)
\end{aligned}
$$

with symmetric positive definite control gain matrices $K_{P}, K_{D}, K_{T}$ and $K_{S}$. The motor position errors of this MIMO state feedback controller without friction compensation (Fig. 9) are worse than the adaptive controller. However the MIMO state feedback controller can achieve a better dynamic behavior that can be shown in a single period of the measured link torques (Fig. 10 and Fig. 11) concerning smaller noise and superior damping in the reversal points of the trajectory. The reason is that the adaptive control scheme use link position, velocity as well as acceleration which the MIMO state feedback controller ignores the acceleration.

For the case of interacting with the environment the adaptive control scheme is not longer suited due to the chattering 

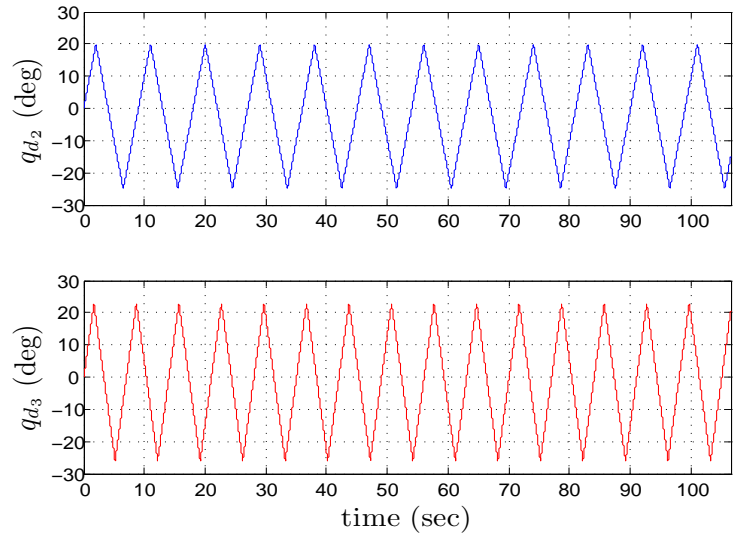

Fig. 3. Desired link position.

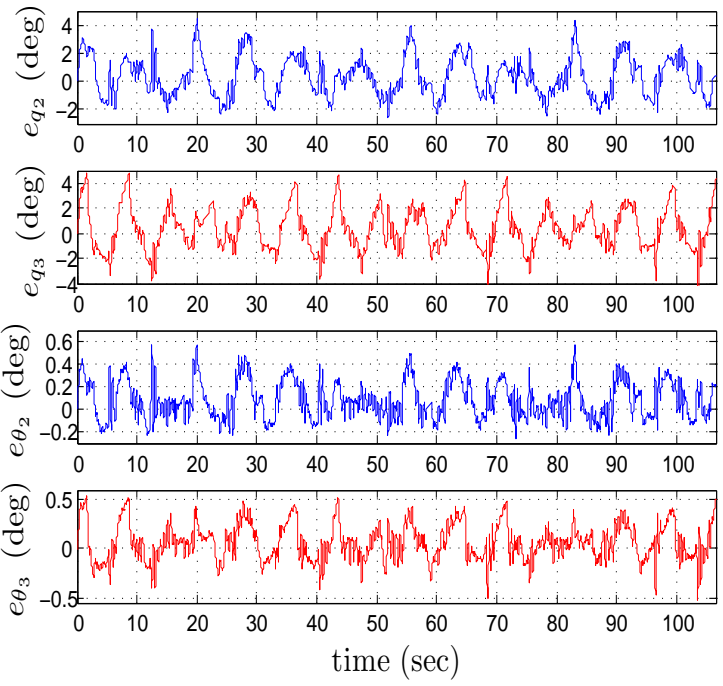

Fig. 4. Tracking link and motor position errors with the computed torque scheme.

phenomena near zero velocity. Then a compensation with the dynamic friction model or with a friction observer [12] is proposed.

\section{CONCLUSIONS}

In this paper we have proposed an adaptive scheme with friction compensation that can be used in order to enhance the robot accuracy. The friction model used in this paper is static, containing Coulomb, viscous and load dependant effects. The static friction parameters are estimated online because of their time variance. Finally, global asymptotic stability of the adaptive controller has been proven. Experimental results validate the approach for the DLR medical robot.

\section{REFERENCES}

[1] U. Hagn, M. Nickl, S. Jörg, G. Passig, T. Bahls, A. Nothhelfer, F. Hacker, L. Le-Tien, A. Albu-Schäffer, R. Konietschke, M. Grebenstein, R. Warpup, R. Haslinger, M. Frommberger, and G. Hirzinger. The DLR MIRO: A versatile lightweight robot for surgical applications. Industrial Robot: An International Journal, pages 324 - 336, 2008.
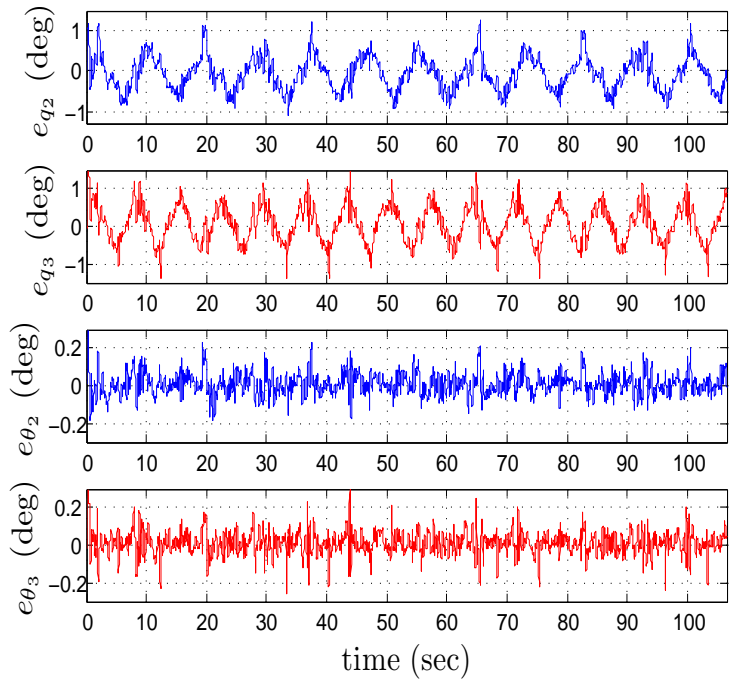

Fig. 5. Tracking link and motor position errors with the adaptive control scheme.
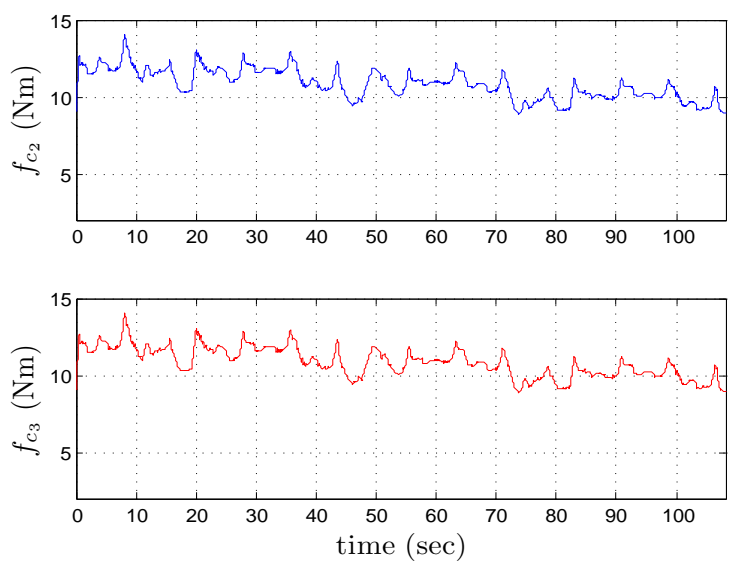

Fig. 6. Estimated Coulomb friction coefficient.

[2] A. De Luca and P. Lucibello. A general algorithm for dynamic feedback linearization of robots with elastic joints. IEEE International Conference on Robotics and Automation, pages 504-510, 1998.

[3] B. Brogliato, R. Ortega, and R. Lozano. Global tracking controllers for flexible-joint manipulators: a comparative study. Automatica, 31:941956, 1995.

[4] L. Tian and A.A. Goldenberg. Robust adaptive control of flexible joint robots with joint torque feedback. IEEE International Conference on Robotics and Automation, pages 1229-1234, 1995.

[5] M.W. Spong. Modeling and control of elastic joint robots. Journal of Dynamic Systems, Measurement and Control, 109:310-319, 1987.

[6] H. D. Taghirad and M. A. Khorsavi. A robust linear controller for flexible joint manipulators. IEEE/RSJ International Conference on Intelligent Robots and Systems, pages 2936-2941, 2004.

[7] P. Tomei. A simple PD controller for robots with elastic joints. IEEE Transaction on Robotics and Automation, pages 1208-1213, 1991.

[8] A. Albu-Schäffer and G. Hirzinger. A globally stable state-feedback controller for flexible joint robots. Journal of Advanced Robotics, pages 799-814, 2001.

[9] L. Le-Tien, A.Albu-Schäffer, and G. Hirzinger. MIMO state feedback controller for a flexible joint robot with strong joint coupling. IEEE International Conference on Robotics and Automation, pages 3824 3830, 2007.

[10] B. S. R. Armstrong. Dynamics for robot control: Friction modeling and ensuring excitation during parameter identification. Dissertation, Stanford University, 1988.

[11] C. C. de Wit, H. Olsson, K. J. Astron, and P. Linschinsky. A new model 

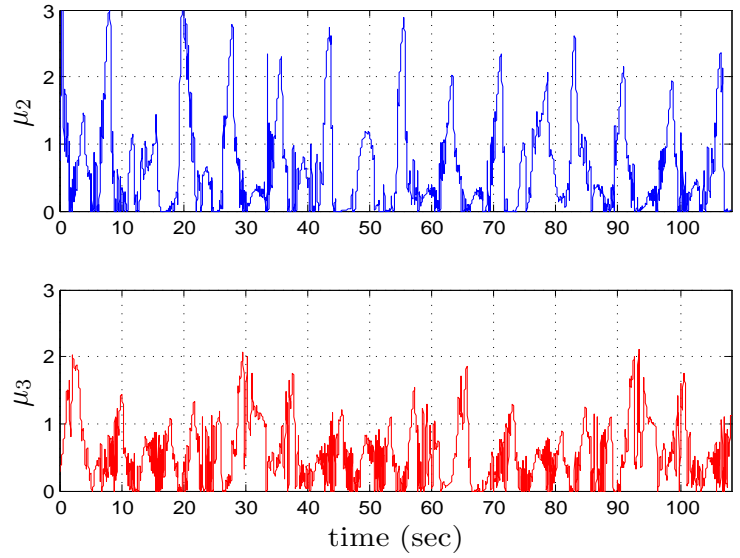

Fig. 7. Estimated load dependent friction coefficient.
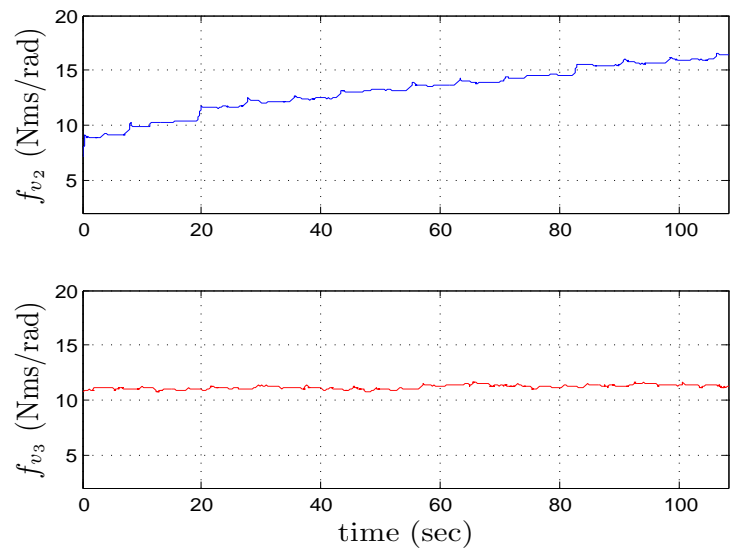

Fig. 8. Estimated viscose friction coefficient.

for control of systems with friction. IEEE Transaction on Automatic Control, 40:419-425, 1994.

[12] L. Le-Tien, A.Albu-Schäffer, A. De Luca, and G. Hirzinger. Friction observer and compensation for control of robots with joint torque measurement. IEEE/RSJ 2008 International Conference on Intelligent Robots and Systems, pages 3789 - 3795, 2008.

[13] C. C. de Wit and P. Lischinsky. Adaptive friction compensation with partially known dynamic friction model. International Journal of Adaptive Control and Signal Processing, 11:65-80, 1997.

[14] P. Tomei. Robust adaptive friction compensation for tracking control of robot manipulators. IEEE Transaction on Robotics and Automation, 45:2164-2169, 2000.

[15] Q. H. Xia, S. Y. Lim, M. H. A. Jr, and T. M. Lim. Adaptive joint friction compensation using a model-based operational space velocity observer. IEEE International Conference on Robotics and Automation, pages 3081-3086, 2004.

[16] Y. Zhu and P. R. Pagilla. Static and dynamic friction compensation in trajectory tracking control of robots. IEEE International Conference on Robotics and Automation, pages 2644-2649, 2002.

[17] J-J. E. Slotine and W. Li. Applied Nonlinear Control. Prentice-Hall International, 1991.
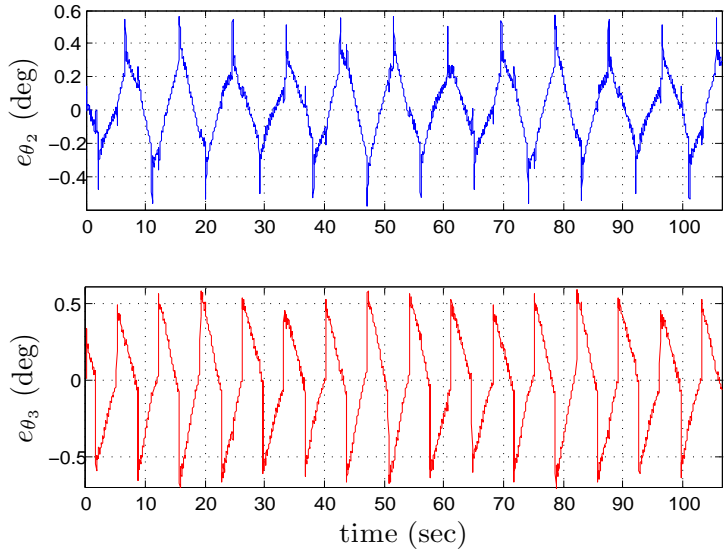

Fig. 9. Tracking motor position errors with the MIMO state feedback controller.
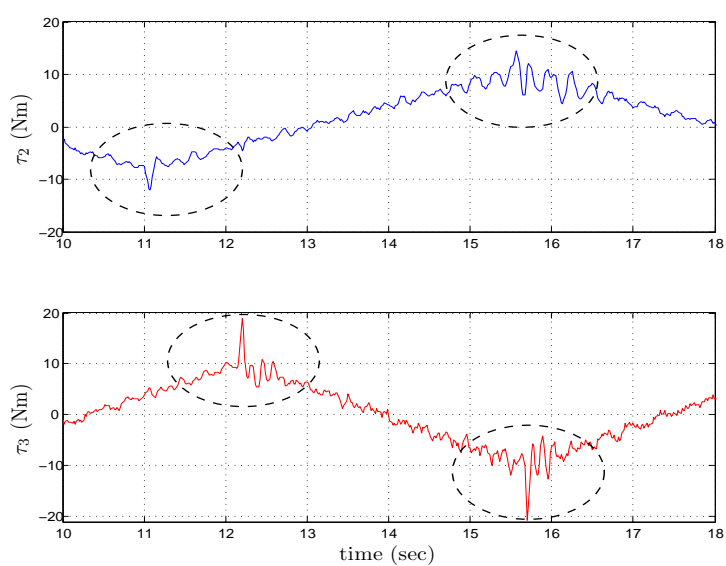

Fig. 10. Measured link torque of the adaptive control scheme.
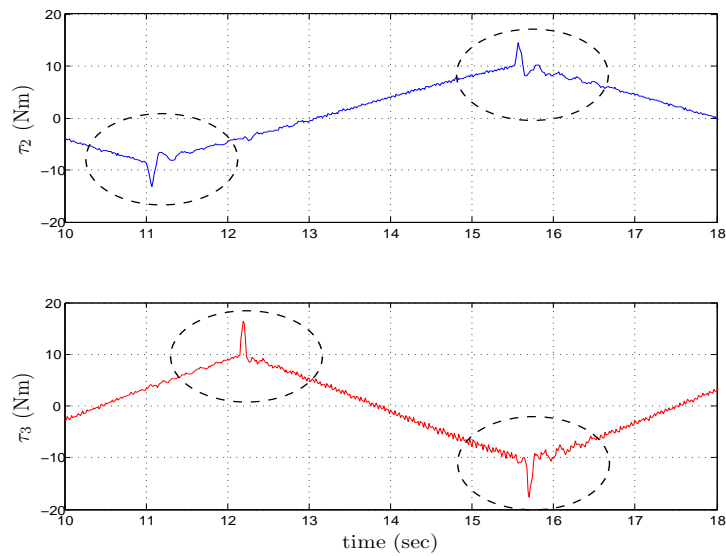

Fig. 11. Measured link torque of the MIMO state feedback control scheme. 